\title{
VON ZAGHAFTEM NACHHALL. ZUR REZEPTIONSGESCHICHTE DER ORATORIEN VON JOHANN JOSEPH FUX
}

I.

„Ich glaube an den einen Gott, den Vater, den allmächtigen, den Schöpfer des Himmels und der Erde, alles Sichtbaren und Unsichtbaren. Und an den einen Herrn Jesus Christus, den Sohn Gottes, einziggeborenen, und aus dem Vater geboren vor aller Zeit. Gott von Gott, Licht von Licht, wahrer Gott vom wahren Gott, gezeugt, nicht erschaffen, gleichen Wesens mit dem Vater, durch den alles geschaffen worden ist." "Jahrhunderte lang haben die Gläubigen dieses, das Nicänische Glaubensbekenntnis gebetet, das auf das 325 abgehaltene Konzil von Nicäa zurückgeht - und Komponisten haben es vertont. Erst im Gefolge des Zweiten Vatikanischen Konzils wurde das kürzere Apostolische Glaubensbekenntnis favorisiert und setzte sich nach und nach in allen Diözesen der Römisch-Katholischen Kirche durch. Anhand dieses Beispiels lässt sich veranschaulichen, wie weit wir heute von manchen Textgrundlagen des Glaubens entfernt sind, die zugleich zu Selbstverständlichkeiten der Musikkultur geworden waren. Wenn die Grenzen zwischen Liturgie einesteils und Andacht andernteils durch Volkssprache, tätige Teilnahme der Gläubigen und auch in der Bevorzugung bestimmten geistlichen Liedgutes im Gottesdienst heute fließender denn je geworden sind, steht dies quer zu jenem musikalischen Erbe, das im Sinne der Constitutio de sacra liturgia von 1964 als „Schatz der Kirchenmusik“ hoch gehalten bleiben soll. ${ }^{2}$

1 „Credo in unum Deum, patrem omnipotentem, factorem coeli et terrae, visibilium omnium et invisibilium. Et in unum dominum Jesum Christum, filium Dei unigenitum et ex patre natum ante omnia saecula. Deum de Deo, lumen de lumine, Deum verum de Deo vero, genitum, non factum, consubstantialem patri, per quem omnia facta sunt.“

2 „[...] Der Schatz der Kirchenmusik möge mit größter Sorge bewahrt und gepflegt werden. [...]." Constitutio de sacra liturgia (Konstitution über die heilige Liturgie), übersetzt und 
Diese einleitenden Gedanken sollen nicht als Kritik verstanden werden; sie möchten nur einer Distanz gewahr werden, die es zu überbrücken gilt, um ein Corpus geistlicher Musik aus dem 18. Jahrhundert mit möglichst geringem Verlust an Einsicht und Verständnis zur Sprache zu bringen. Oratorien gehörten bis zum Zweiten Vatikanischen Konzil zur ,pia exercitia', die Andachten und Prozessionen bündelte, aber - getreu ihrem Namen - eben auch als ,exercitium', als tätige Übung zu verstehen war, der überdies notwendiger Weise eine innere Erneuerung im Geiste der ,re-ligio“ vorausging. Im Gegensatz zur Liturgie war die Sprachwahl hier offen - auch die Volkssprache konnte verwendet werden. ${ }^{3}$ Fux' Oratorienschaffen entspricht dieser Gegebenheit insofern, als sich darunter ein „Oratorium germanicum“ über deutschem Text befindet (wenngleich sich über dessen Zuschreibung an den Wiener Hofkapellmeister disputieren lässt ${ }^{4}$ ).

Alle anderen überlieferten Oratorien Fux' entstanden indes für Aufführungen in der Kapelle des kaiserlichen Hofes in Wien. Die dort jährlich in der Fastenzeit mehrmals gegebenen Oratorien, in der Regel verschiedenen Komponisten anvertraut, unterschieden sich durch ihre italienische Sprache sowohl von den Volksandachten als auch von der seit dem Mittelalter zur Gestaltung des Osterfestes mehr und mehr herausgearbeiteten ,Klerusliturgie“ mit zahlreich vorgeschriebenen, selbstredend lateinischen Lesungen und Gebeten. ${ }^{5}$ Da die Oratorien - wie die auf die gesamte Karwoche verteilten Aufführungsdaten zeigen - dem ,Triduum sacrum' des gekreuzigten, begrabenen und auferweckten Herrn nicht inkorporiert waren, gab es Raum für künstlerische Entwicklung, vergleichbar keiner anderen Gattung geistlicher Musik - und diese Option wurde in Dichtung wie Komposition gleichermaßen genützt. Es greift aber zu kurz, wenn vielfach zu lesen steht, dass das Oratorium in der Fastenzeit ein Ersatz für die Oper gewesen sei. Schon die Tatsache, dass auf Opernaufführungen bei Hoftrauer verzichtet wurde, Oratorien hingegen an die Fastenzeit gebunden waren, zeigt eine grundverschiedene Ausrichtung auf. Demgemäß bilden Oratorien nicht die noch zulässige geistliche Variante säkularer Kulturleistung, sondern eine eigenständige Rubrik im musikalischen Hofkalendarium.

Darüber hinaus war den Oratorien ein spezieller Platz im dynastischen Selbstverständnis zugewiesen. Einesteils waren sie der habsburgischen Virtus unterstellt, worin Pietas, der Frömmigkeit, als Brücke zwischen weltlichen und geist-

kommentiert (durch Josef Andreas JUNGMANN SJ), in Das Zweite Vatikanische Konzil, Freiburg - Basel - Wien 1966 (Lexikon für Theologie und Kirche, Ergänzungsband), Abschnitt „De Musica sacra“ („Die Kirchenmusik“), S. 94-101: 95.

3 TRUMMER, Johann. Das Heilige Grab und die Sepolcro-Komposition. In Johann Joseph Fux: Il fonte della salute, vorgelegt von Martin JIRA, Graz: ADEVA 2008 (Johann Joseph Fux. Sämtliche Werke IV/5), S. XV-XXIII: XVI.

4 Diesbezüglich äußerten sich Guido ERDMANN und Herbert SEIFERT im Rahmen der Diskussion zu diesem Referat.

5 TRUMMER (wie Anm. 3), S. XVI 
lichen Tugenden eine tragende Funktion zukam. ${ }^{6}$ Andererseits sorgte der private Rahmen der Hofburgkapelle für jenes Bekenntnis zur persönlichen Verinnerlichung des Glaubens, das die ,pia exercitia' voraussetzte. Doch auch diese Privatheit ist Maske. Keiner der Textdichter von Fux nimmt explizit Bezug auf persönliche Belange des Kaisers bzw. der Mitglieder des Herrscherhauses oder traute sich gar Fehlverhalten zu rügen. Selbst Kaiser Joseph I., dessen Liebesleben insbesondere nach dem Tod des Vaters von reichhaltiger Abwechslung geprägt war? durfte wissen, dass alle seine Fehltritte tabu bleiben würden.

Umso erstaunlicher mutet angesichts dieser, dynastischen ' Lesart von Privatheit aber dann zunächst an, dass man in der Hofburgkapelle ein Heiliges Grab errichtete (wie es sich aus mittelalterlichen Anfängen heraus im 15. und 16. Jahrhundert zur Gewohnheit entwickelt hatte). Eine derartige Anpassung an die Religiosität im Volk passt durchaus nicht ins gedankliche Konzept der privaten höfischen Andacht, wogegen Öffentlichkeitswirkung bei anderen Anlässen - so etwa, wenn Kaiserin Eleonora 1697 die Ikone Maria Pocs mit einer diamantenen Rose schmückte ${ }^{8}$ oder

Der spezifisch habsburgische Tugendkodex setzte sich aus den weltlichen Tugenden Fortitudo, Clementia, Liberalitas, Pietas, Justitia und Modestia und den christlichen Tugenden Fides, Spes und Caritas zusammen. Siehe dazu zusammenfassend HILSCHER, Elisabeth Theresia. Der Kaiserliche Hofpoet Pietro Metastasio (1698-1782). Zur Kulturgeschichte des Hofes Kaiser Karls VI. Graz: Johann-Joseph-Fux-Gesellschaft 2000 (Jahresgabe der Johann-Joseph-Fux-Gesellschaft 23), S. 5, sowie zum Hintergrund CORETH, Anna. Pietas Austriaca. Österreichische Frömmigkeit im Barock. 2. Aufl. Wien: Verlag für Geschichte und Politik 1982 (Österreich-Archiv. Schriftenreihe des Instituts für Österreichkunde o. Zl.).

Im Übrigen starb Joseph 1711 in den Armen seiner Geliebten, Gräfin Marianne Pálffy. Vgl. MRAZ, Gerda. Die Kaiserinnen aus dem Welfenhaus und ihr Einfluß auf das geistig-kulturelle Leben in Wien. In Johann Joseph Fux und seine Zeit. Kultur, Kunst und Musik im Spätbarock, hg. v. Arnfried EDLER und Friedrich Wilhelm RIEDEL. Laaber: Laaber 1996 (Publikationen der Hochschule für Musik und Theater Hannover 7), S. 75-91: 76. Am Beispiel der Kaiserinnen Amalie Wilhelmine (1673-1742, seit 1711 Witwe nach Joseph I.) und Elisabeth Christine (1691-1750, seit 1740 Witwe nach Karl VI.) zeigt Mraz auf, wie private Religiosität im Kaiserhaus greifen konnte, indes - dies meine Beobachtung - zugleich doch jenen karitativen Zug annahm, der auch heute das Wirken gekrönter wie ungekrönter Staatsoberhäupter begleitet.

Die Übertragung dieser ungarischen Ikone war auf Wunsch der Kaiserin, Gemahlin Leopolds I., veranlasst worden. Zunächst wurde das Gnadenbild sodann in der Favorita, der kaiserlichen Sommerresidenz (dem heutigen Theresianum) aufgestellt, am 7. Juli 1697 schließlich in die Augustiner-Hofkirche übertragen. Dort zierte die Kaiserin selbst das Gnadenbild mit einem kostbaren Geschmeide, einer diamantenen Rose, was den Beinamen „Rosa mystica“ entstehen ließ. Danach befand sich das Bildnis 14 Tage lang zur Anbetung in St. Stephan, ehe es in die Favorita zurückgebracht wurde. „Weil aber alle christlichen Gemeinden ein inbrünstiges Verlangen äußerten, dieses Gnadenbild in ihren Kirchen verehren zu können" wie es in einer frühen Beschreibung heißt - stellte man die Ikone anschließend in verschiedenen Wiener Kirchen aus, ehe sie am 1. Dezember 1697 endgültig nach St. Stephan übertragen und dort bis zum Ende des Zweiten Weltkriegs auf dem Hochaltar aufgestellt war. Vgl. dazu HOCHRADNER, Thomas -VÖRÖSMARTY, Géza Michael. Zur Musikpflege am Altar Mária Pócs (Maria Pötsch) in St. Stephan in Wien. Studia Musicologica Academiae Scientiarum Hungaricae 2000, Jg. 41, Heft 1-3, S. 133-175. 
der Kaiser jährlich das Heilige Grab in der Wiener Universitätskirche besuchte ${ }^{9}$ durchaus instrumentalisiert wurde. Was seitens der Gegenreformation als Fokus einer veranschaulichten Glaubensdarstellung stark forciert worden war ${ }^{10}$, bedurfte ja des öffentlichen Gestus - erst daraus erklärt sich die Eignung der Szenerie als Anlass theatralischer Darstellungen. Entscheidend ist nun aber, dass diese Theatralität für den kaiserlichen Hof offenbar reizvoll genug war, um ihre Öffentlichkeit in eine eigene Form der Privatheit zurückzuholen, eine künstlerische Privatheit, ausgetragen mit hoher poetischer Qualität, manifestiert in der 1657 unter Ferdinand III. gegründeten literarischen Akademie und über Generationen hinweg als primär poetische, sekundär musikalische Gattung weiter geführt und entwickelt. ${ }^{11}$

Zwei Meldungen im Wienerischen Diarium von 1714 zeigen die Unterschiede im Öffentlichkeitscharakter der Aufführungen am Wiener Hof auf: „Heut | Abends haben die Röm. Kaiserl. und Königlich=Catholische Majestät $\mid$ in Begleitung des Päbstl. Herrn Nuntius, und des Venetianischen Herrn Bottschafters | dan viel=anderer hohen Stands=Personen $\mid$ in der Kaiserl. Hof=Kirchen deren W.W. E.E. P.P. Augustiner Barfüssern | bey einer mit künstlicher Beleuchtigung geziehrt=Geistlicher Vorstellung | und anmüthiger Musick | dem GOttesdienst [...] höchst=andächtigst beygewohnet" heißt es über die Aufführung eines Oratoriums in der Augustinerkirche, ,sodan wieder des Abends in gemeldter Capellen [der Kaiserlichen Hofburgkapelle] bey dem Wälschen Oratorium und Predig mit dem gewöhnlichen Gefolg Sich eingefunden“" wenig später zu einem Oratorium, das in der Hofburgkapelle geboten wurde. ${ }^{12}$ Gelegentlich verdeutlichen solche Notizen überdies, dass die Aufführungen in den Frömmigkeitskontext des kaiserlichen Hofes eingebunden waren: „Nach geendigter Andacht kamen Ihre Kaiserl. Majestät zu Mittag in Dero Leib=Wagen wieder zuruck in die Kaiserl. Burg $\mid$ alwo Abends Ihre Majestät in der Kaiserl. Hof=Capelle der Italiänischen Predig | als auch dem gesungenen Italiänischen Oratorio unter dem Titel: Christus im Garten (welches von Herrn Pariati Kaiserl. Poëten componirt | und von Herrn Johann Joseph Fuchs kaiserl. Capell=Meister in die Music gebracht worden) und dann letztlichen der

9 ADEL, Kurt. Das Jesuitendrama in Österreich. Wien 1957, S. 17, zit. nach GRUBER, Gernot. Das Wiener Sepolcro und Johann Joseph Fux. 1. Teil [ein zweiter Teil ist nicht erschienen]. Graz: Johann-Joseph-Fux-Gesellschaft 1972 (Jahresgabe der Johann-Joseph-Fux-Gesellschaft 9), S. 18.

10 Vgl. dazu TRUMMER (wie Anm. 3), S. XVII.

11 Zum Kunstcharakter der für den Wiener Hof komponierten Oratorien siehe weiterführend GRUBER (wie Anm. 9), S. 16-20.

12 Wienerisches Diarium 1714, Nr. 1109, 17. 3. 1714, bzw. Wienerisches Diarium 1714, Nr. 1110, 23. 3. 1714; welche Oratorien jeweils zur Aufführung kamen, lässt sich nicht exakt feststellen. In Frage kommen hier La fede sacrilega nella morte del precursor S. Giovanni Battista von Johann Joseph Fux, Santa Flavia domitilla von Antonio Caldara und L'umiltà coronata in Ester von Antonio Lotti. 
Litaney beygewohnet." wird im Wienerischen Diarium für den Dienstag der Karwoche 1723 berichtet. ${ }^{13}$

\section{II.}

Zusammengefasst: Für Aufführungen durch die Wiener Hofmusikkapelle in der vorösterlichen Fastenzeit komponierte Johann Joseph Fux zwischen 1702 und 1728 insgesamt zwölf Oratorien. Alle diese Werke erklangen erstmals in der Hofburgkapelle, sechs davon in halbszenischer Umsetzung als ,Sepolcri' vor dem in der Karwoche im Kirchenraum errichteten Heiligen Grab (vgl. Tab. 1). ${ }^{14}$ Fünf Oratorien (La donna forte, Il fonte della salute, Cristo nell'orto, Il testamento di nostro Signor Gesù Cristo sul Calvario und La deposizione dalla croce di Gesù Cristo Salvator nostro) wurden in einem späteren Jahr nochmals aufgeführt. Aufführungstag für die Oratorien war meistens der Gründonnerstag, selten der Dienstag der Karwoche, die Sepolcri wurden zunächst am Karfreitag, dann vor allem am Dienstag der Karwoche gegeben. Unter den Textdichtern dominiert Pietro Pariati (1665-1733) $)^{15}$ mit insgesamt sieben Libretti, je einmal griff Fux auf Dichtungen von Bernardino Maddali (Nachweise 1716-1727), Giovanni Claudio Pasquini (1695-1763), Pier Maria Ruggieri (Lebensdaten unbekannt) und (vermutlich) Giovanni Andrea Lorenzani (1637-1712) zurück. Für eines der Oratorien ist der Librettist nicht bekannt (vgl. Tab. 1).

Breit gestreut ist der thematische Rahmen der fuxschen Oratorien: Er umfasst alt- und neutestamentliche Stoffe ebenso wie hagiographische und auf biblischen, manchmal auch sonstigen religiös-kontemplativen Texten beruhende allegorische Handlungen. Eine Tabelle (s. Tab. 1) verdeutlicht einesteils, dass Fux mit dem seit 1714 als ,kaiserlicher Kammerdichter' am Wiener Hof beschäftigten Pietro Pariati von dessen Bestellung an bis 1720 bevorzugt zusammengearbeitet hat, und andernteils, dass er sich mit den Jahren zunehmend auf ,Sepolcro'-Oratorien über Sujets aus dem Neuen Testament konzentrierte.

Da der Fundus der Wiener Hofmusikkapelle sorgfältig verwaltet wurde, kam es nur selten zu einer Weitergabe des Notenmaterials. Doch gelangten eine zeitgenössische Partitur von La Regina Saba anonym nach Schwerin ${ }^{16}$ sowie Partituren von Il fonte della salute, Il testamento di nostro Signor Gesù Cristo sul Calvario und

Wienerisches Diarium 1723, Nr. 24, 24. 3. 1723, zum am Dienstag der Karwoche, dem 23. März 1723 wiederholten Sepolcro Cristo nell'orto von Johann Joseph Fux.

Zur Frage, ob dem ,Sepolcro“ der Rang einer eigenständigen Gattung zuzusprechen sei, s. GRUBER (wie Anm. 9) sowie den Beitrag von Herbert SEIFERT im vorliegenden Heft.

Vgl. GRONDA, Giovanna (Hg.). La carriera di un librettista. Pietro Pariati da Reggio di Lombardia. Reggio Emilia: Il Mulino 1990 (Proscenio. Quaderni del Teatro Municipale »Romolo Valli« di Reggio Emilia 5). 
La deposizione dalla croce di Gesù Cristo Salvator nostro - wie auch etliche weitere, vor allem dramatische Werke aus Wien betreffende Abschriften - auf Betreiben des regierenden Herzogs Anton Ulrich von Sachsen-Meiningen (1687-1763) nach Meiningen. Dieser Herzog, der sich seit 1724 in Wien aufgehalten hatte, ließ in kaum nachvollziehbarem Sammeleifer eine Unzahl von Partituren aus dem Fundus der Wiener Hofmusikkapelle durch Hofkopisten abschreiben. ${ }^{17}$ Andere Überlieferungen stehen dagegen außerhalb von Liebhaberei und Hortung. Die Sinfonia zu La fede sacrilega wurde als ,Sonata a 4' verwendet - zunächst vermutlich innerhalb der privaten Musikkapelle der Kaiserinwitwe Amalie Wilhelmine; später gelangte eben dieses Notenmaterial ins Stift Kremsmünster. ${ }^{18}$ Zwei Quellen aus dem 18. Jahrhundert überliefern die Sinfonia außerdem als Sonata für Tasteninstrument. Auch die Madrigale aus La donna forte und Il disfacimento di Sisara sind in mehreren, und zwar später zu datierenden Quellen erhalten. Sie geben ein offenkundiges Zeugnis der Wertschätzung, die man Fux als Meister des Kontrapunktes entgegenbrachte.

Eine interessante sekundäre Überlieferungsebene erhielt das Sepolcro Cristo nell'orto infolge einer für Salzburg in den 1720er Jahren zu vermutenden Aufführung. Deren Partitur wurde in neuerlich leichter Umarbeitung zur Vorlage einer Aufführung in Brno (Brünn), die mittels eines 1731 ebenda bei Swoboda aufgelegten Textdrucks gesichert ist. Die Abschrift gelangte als Bestandteil der Sammlung Voß in die Musikabteilung der Deutschen Staatsbibliothek Berlin. Ein vergleichbares ,Schicksal' nahm La deposizione dalla croce di Gesù Cristo Salvator nostro; auch dazu sind in Salzburg und Berlin (Sammlung Voß) Partituren erhalten und ist, in diesem Fall für 1729, eine Aufführung in Brno (Brünn) durch einen bei Swoboda publizierten Textdruck belegt.

Großherzoglichen Mecklenburg-Schweriner Fürstenhauses. 2 Bände, Schwerin 1893, Nachdruck Hildesheim - New York: Olms 1974. Bd. 1, Anonyma Nr. 10, S. $6 f$.

17 Über die Ankäufe informieren zwei Erwerbslisten: Catalogus / von / Gebundnen und Ungebundnen / Büchern, / so Anno 1728 aus Wien mit / zurückgebracht worden. (Meiningen, Thüringisches Staatsarchiv, GAXV. T. 47) sowie Catalogus / von / gebundnen und ungebundnen / Musicalien / so Anno 1728 von Wien mit / zurückgebracht worden. (Meiningen, Thüringisches Staatsarchiv, GAXV. T. 49, fol. 291-308), ferner die Tage- und Rechnungsbücher Herzog Anton Ulrichs aus den Jahren 1726 und 1727 (Meiningen, Thüringisches Staatsarchiv, GA XV. T. 54). Vgl. darüber hinaus BENNETT, Lawrence E. A little-known Collection of early-eighteenthcentury Vocal Music at Schloss Elisabethenburg, Meiningen. Fontes Artis Musicae 2001, Jg. 48, S. 250-302.

18 ERDMANN, Guido. Die Sonaten für größere Besetzungen von Fux (K 343-350) und die Frage nach ihren zeitgenössischen Kontexten. Referat bei der Tagung Werk und Wirkung? - Johann Joseph Fux zum 350. Geburtstag. Wien 2010, Druck in Vorb.; ders., Vorwort zur Edition der Sinfonia in Bd. VI/6 der Ausgabe sämtlicher Werke von Johann Joseph Fux, in Vorb. Für die vorab gewährte Einsicht in diese Texte, ebenso wie für die kollegiale Zusammenarbeit über Jahre hinweg dankt der Verfasser Guido Erdmann sehr herzlich. 


\section{III.}

Ein anderer Überlieferungsweg führt nach Böhmen. Im Kloster der Kreuzherren mit dem Roten Stern in Prag wurde 1737/38 das Musikalieninventar Musicalium Gayerianorum omnium angelegt, um die nach dem Ankauf der privaten Musikaliensammlung des verstorbenen Kapellmeisters von St. Veit in Prag, Christoph Karl Gayer (?1668-1734) stark angewachsenen Bestände besser zu übersehen. Auf den letzten Blättern des Inventars finden sich etliche Einträge, Oratorien - darunter mehrere von Johann Joseph Fux - betreffend („Opera, \& Oratoria descripta“ bzw. „Partiturae ab Oratorijs non restitutae“ überschrieben). Demnach verfügte man über Partituren zu La fede sacrilega, La donna forte, Il fonte della salute, Il disfacimento di Sisara, Cristo nell'orto und Gesù Cristo negato da Pietro. ${ }^{19}$ Diese Materialien sind verloren gegangen, für 1750 und 1751 durch Textdrucke aber zwei aus verschiedenen Werken kompilierte Oratorienaufführungen in der Klosterkirche bekannt, bei denen einmal gewiss, andermal vielleicht auf Musik von Johann Joseph Fux zurückgegriffen wurde: Gesù Cristo negato da Pietro $(1750)^{20}$ und Oratorium vel compamento collectum ex diversis Authoribus titulatur la Deposizione dalla Croce prima pars coram magna Nobilitate productum à Bleileb ${ }^{21}$ (1751).

Über das Musikalieninventar zum Bestand des Klosters der Kreuzherren mit dem Roten Stern in Prag besteht auch eine Verbindung zu jenem fuxschen Oratorium, das nur außerhalb des Wirkungskreises der Wiener Hofmusikkapelle nachzuweisen ist: dem „Oratorium germanicum“" ${ }^{\text {“22 }}$, das in zwei Quellen, einem

19 Zu den Oratorienaufführungen in der Kirche des Klosters der Kreuzherren mit dem Roten Stern in Prag s. FUKAČ, Jiř́. Die Oratorienaufführungen bei den Prager Kreuzherren mit dem Roten Stern als Typ lokaler Musikfeste. Sborník Prací Filozofizké Fakulty Brněnské Univerzity 1994, Jg. 29, S. 69-89; ders., Pöltenberg, Wien und die Musikkultur der Prager Kreuzherren. In Österreichische Musik - Musik in Österreich. Beiträge zur Musikgeschichte Mitteleuropas. Theophil Antonicek zum 60. Geburtstag, hg. v. Elisabeth Theresia HILSCHER. Tutzing: Schneider 1998 (Wiener Veröffentlichungen zur Musikwissenschaft 34), S. 171-177.

20 Notiz im Musikalieninventar der Kreuzherren mit dem Roten Stern, Nachträge fol. 56 ${ }^{\mathrm{v}}$, Nr. 14: „Anno 1750 Oratorium collectum secunda pars libelli Giesù negato ex diversis Autho- | ribus Fux, Contini, Graun, Porpora, Lotti cum applausu productum à Bleileb.“

21 Joseph Bleylebel (gest. 1762), Regens chori im Prager Kloster der Kreuzherren mit dem Roten Stern. Bleylebel dürfte jeweils selbst die Kompilation vorgenommen haben. Noch ein weiteres kompiliertes Oratorium mit dem Titel La Sepoltura lässt sich anhand des Musikalieninventars der Kreuzherren mit dem Roten Stern, fol. 56 velegen. Vgl. FUKAČ, Die Oratorienaufführungen bei den Prager Kreuzherren (wie Anm. 19), S. 84.

Zum „Oratorium germanicum“ vgl. PETERMAYR, Klaus. Fux oder Caldara? - zum wiederaufgefundenen >Oratorium Germanicum< (E 61). Auf Fux-Jagd 2004, Jg. 12, Nr. 1/2, S. 23-32; ders., Das Oratorium Germanicum (E 61) von Johann Joseph Fux. Jahrbuch des rism-österreich 2011, Jg. 2 (Veröffentlichungen des rism-österreich A 16), S. 79-91, und zuletzt FLOTZINGER, Rudolf. Das 〉Oratorium Germanicum de Passione` von Johann Joseph Fux. Schritte der Annäherung an Autoren- und Gattungsfragen. In Festschrift Hellmut Federhofer zum 100. Geburtstag, hg. v. Axel BEER in Verb. mit Gernot GRUBER und Herbert SCHNEIDER. Tutzing: Schneider 2011 (Mainzer Studien zur Musikwissenschaft 45), S. 91-105. 
Fux zugeschriebenen, mit 1731 datierten handschriftlichen Textbuch im Stift Kremsmünster ${ }^{23}$ und einem Antonio Caldara zugewiesenen, undatierten Stimmensatz im Kloster Ottobeuren ${ }^{24}$ überliefert ist. Als Autor der mythologisch-allegorischen Handlung (nach Apollodor, Mythologische Bibliothek II, 42-44) vermutet Rudolf Flotzinger den aus Hamburg gebürtigen, in Wien sesshaft gewordenen Heinrich Rademin (1674-1731). ${ }^{25}$ Zwei Indizien sprechen für eine Autorschaft Fux' am „Oratorium germanicum“: zum einen die Solidität in der Vertonung eines deutschen Librettos, zum anderen der Umstand, dass für die zahlreichen im Stift Kremsmünster vorhandenen Quellen teils eine direkte Verbindung nach Wien nachgewiesen werden kann, während der entsprechende Bestand im Musikarchiv des Klosters Ottobeuren, nicht zuletzt aufgrund der erst um 1750 anzusetzenden Entstehungszeit der Abschriften, in Hinsicht auf die Fux in den Umschlagtiteln zuerkannte Urheberschaft der Werke bei unikaten Überlieferungen durchaus zu Zweifel Anlass gibt. Caldara darf somit als Autor des Werkes ausgeschieden werden; Fux' Urheberschaft wird aber dadurch nicht bestätigt, sondern im Gegenteil durch einen Eintrag im Musikalieninventar der Kreuzherren in Frage gestellt, das unter den „Opera, \& Oratoria descripta“ als Nr. 6 ein „Oratorium Germanicum die Von Perseo Verfreüte [vermutlich: befreite] Andromeda ex rebus defuncti Geyer a 5 voc [i]“" anführt. ${ }^{26}$ Die genannten Personen der Handlung entsprechen dem „Oratorium germanicum“ und ermöglichen eine Identifizierung zumindest der Textgrundlage. Ein Komponist wird aber nicht genannt; infolge des Vermerks „diversimodè Compilatum“ ist von einem Pasticcio-Werk auszugehen.

Jiří Fukač - seinen Namen erwähne ich mit großem Respekt und in dankbarer Erinnerung - hat der Musikpflege der Kreuzherren in Prag und Pöltenberg bei Znaim eine Reihe von Studien gewidmet, die mir aus sprachlichen Gründen leider nicht alle zugänglich sind. Darunter befindet sich mit Die Oratorienaufführungen bei den Prager Kreuzherren mit dem Roten Stern als Typ lokaler Musikfeste $e^{27}$ ein Aufsatz in deutscher Sprache, der in bestechender Weise dem Phänomen gelenkter und zugleich im Sinne eines eigenen kulturellen Traditionsbewusstseins gestalteter musikalischer Frömmigkeit in der Prager Klosterkirche zum Heiligen Franz Seraph nachspürt. Fukač knüpft daran die Frage eines ,lokalen Musikfestes sui generis“ und beantwortet sie unter Bedacht auf verschiedene Prager Musikanlässe letzten Endes positiv. Er verweist insbesondere auf die Abhaltung von Schiffsprozessionen mit Musik zu Ehren des Heiligen Johann Nepomuk an der Karlsbrücke (an deren

23 Geschrieben von Ignaz Dansky (1717-1797), der seit 1736 als Musiker und Kopist für das Stift Kremsmünster tätig war, datiert mit „1731“; s. dazu FLOTZINGER (wie Anm. 22), S. 98. 1737 seine Primiz gefeiert hatte; s. dazu PETERMAYR, Das Oratorium Germanicum (wie Anm. 22), S. 81.

25 FLOTZINGER (wie Anm. 22), S. 103f.

26 FUKAČ, Die Oratorienaufführungen bei den Prager Kreuzherren (wie Anm. 19), S. $82 f$.

27 Vgl. Anm. 19. 
Altstädter Ufer die Kirche der Kreuzherren unmittelbar gelegen ist), weiters auf die im Böhmen der 1720er und 1730er Jahre stark anwachsende Zahl von Oratorienaufführungen und das Vorbild der Musikdarbietungen anlässlich der Feierlichkeiten zur Krönung Karls VI. zum König von Böhmen $1723 .{ }^{28}$ Wesentlich trägt zu Fukačs Einschätzung bei, dass die Pflege von Oratorien in der Prager Kreuzherrenkirche sowohl in der Wahl des Repertoires (mit Werken auch aus Italien und Mitteldeutschland) als auch infolge der breiten Resonanz eine einzigartige Stellung und damit eine Art ,Pilotfunktion“ für die Musikpflege der böhmischen Länder insgesamt einnimmt, ehe ab den 1770er Jahren der Wunsch nach Aufführung von Werken böhmischer Komponisten das Interesse an Musik aus ,ferneren' Ländern überwuchs. ${ }^{29}$ Bis 1782 dauerte die Oratorienpflege der Prager Kreuzherren an, ehe sie im Zeichen der kirchlichen Aufklärung ein abruptes Ende nahm. ${ }^{30}$

Damit tritt jener einschneidende Schnitt zutage, der durch die restriktiven Maßnahmen im Sinne der kirchlichen Aufklärung in Bezug auf theatralische Darbietungen gesetzt wurde. Das neue Regulativ griff geradezu brutal in eine Lebenswelt ein, dessen öffentlichkeitscharakter in Teilen in Musik verankert war (ja sogar aus dem sakralen Raum ausgestrahlt hatte, als - nachweislich in den Jahren 1749, 1763 und 1765 - auch Prager Theater während die Karwoche Oratorien anzusetzen begannen, während die Aufführungen in Prager Kirchen stets am Karfreitag stattfanden ${ }^{31}$ ). Darüber hinaus hatte der Orden der Kreuzherren mit dem Roten Stern ein Netzwerk des Musiktransfers über die habsburgischen Länder Böhmen, Mähren, Schlesien und Ungarn gewoben, mittels dessen in der Weitergabe von oratorischen Partituren zugleich für eine gewisse Verbindlichkeit in der Ausübung eines Frömmigkeitsaktes gesorgt war. ${ }^{32}$ Doch mit einem Mal blieb ein Fixpunkt des österlichen Geschehens verwaist, umso mehr, als zudem die übliche Prozession mit dem Allerheiligsten vom Heiligen Grab zum Hochaltar samt ihrem Abschluss mit Te Deum und Regina coeli am Karsamstag Nachmittag entfiel. ${ }^{33}$ Nunmehr galt einzig die liturgische, seit der Ausgabe des Missale Romanum von 1570 verbindlich auf den Vormittag des Karsamstags

28 FUKAČ, Die Oratorienaufführungen bei den Prager Kreuzherren (wie Anm. 19), S. 76-79.

29 Ibid., S. 86-88. Zum Hintergrund vgl. nunmehr BACCIAGALUPPI, Claudio. Rom, Prag, Dresden. Pergolesi und die Neapolitanische Messe in Europa. Kassel et al.: Bärenreiter 2010 (Schweizer Beiträge zur Musikforschung 14).

30 KAMPER, Otaker. Hudebni Praha v XVIII. věku. Prag 1936, S. 252f., zit. nach FUKAČ, Die Oratorienaufführungen bei den Prager Kreuzherren (wie Anm. 19), S. $85 f$.

31 Bei den Kreuzherren entweder um 11 Uhr oder nachmittags, bei den nahen Jesuiten abends; s. dazu FUKAČ, Die Oratorienaufführungen bei den Prager Kreuzherren (wie Anm. 19), S. 79 f. FUKAČ, Pöltenberg, Wien und die Musikkultur der Prager Kreuzherren (wie Anm. 19), S. 174.

33 Bereits im Spätmittelalter hatte sich eingebürgert, das Kreuz (oder eine Christus-Figur, oder das Allerheiligste) am Karfreitag nach einer Prozession ins Grab zu legen und es am Abend des Karsamstag in einer weiteren Prozession wieder zurückzuholen. Siehe dazu GRUBER (wie Anm. 9), S. 8. 
vorgezogene Osterfeier. ${ }^{34}$ Zugleich wurde damit in Gebieten katholischen Glaubens jene Distanz zu oratorischen Musikwerken gelegt, die schließlich ihre Wiederentdeckung im Zeichen des musikalischen Historismus ermöglichte.

\section{IV.}

Obgleich das Nachleben der Werke im Vergleich mit anderen Komponisten spärlich wirkt: Die Rezeption der fuxschen Oratorien noch nach seinem Tod ist in ihrer Intensität außergewöhnlich und wird nur durch seine Messen und Motetten im A-cappella-Stil (und hier in Sonderheit jener beiden, die in den Gradus ad Parnassum veröffentlicht waren ${ }^{35}$ ) überboten. Noch im 19. Jahrhundert war Fux als Komponist von Oratorien durch die Aufnahme des Chores „Tremo la terra" (Nr. 2) aus La deposizione dalla croce in Band 2 der Sammlung vorzüglicher Gesangstücke präsent, den Friedrich Rochlitz (1769-1842) bei Schott in Mainz, Paris und Antwerpen herausgebracht hatte. ${ }^{36}$ Eben dieses Oratorium war durch insgesamt vier private, teils verkürzte Aufführungen in den Hauskonzerten des Wiener Musikliebhabers Raphael Georg Kiesewetter (1773-1850) ins allgemeine Bewusstsein der historistischen Musikszene gelangt. ${ }^{37}$ Ein im hinteren Einbanddeckel einer eigenhändigen Partitur eingeklebtes Blatt fasst die Änderungen zusammen: Kiesewetter verzichtete auf einige Nummern gänzlich, strich außerdem bei Rezitativen und kürzte in nur mit Continuo begleiteten Arien das Da-Capo weg. Die ausschnitthafte Aufführung von La deposizione dalla croce fand vermutlich erst 1842 statt; denn die erwähnte, bei Weitem nicht vollständige Partitur wird in Kiesewetters Galerie der alten Contrapunctisten mit dem Zusatz ,Introduzione ed Arie dell'Oratorio La Deposizione della Croce de G. C. Vienna 1724. Aufgeführt

34 Vgl. TRUMMER (wie Anm. 3), S. XVI. Zur nachkonziliaren Entwicklung vgl. SCHINDLECKER, Katrin. Musik in der Osterliturgie im 20. Jahrhundert. Eine generelle Beobachtung mit zwei Fallbeispielen aus der heutigen Zeit. Diplomarbeit Universität Mozarteum Salzburg, 2008.

35 Offertorium „Ad te Domine levavi“ WV IV.121 (Gradus ad Parnassum, S. 247-254) und Offertorium „Ave Maria“ WV IV.128 (Gradus ad Parnassum, S. 256-261).

36 Johann Joseph Fux, La Deposizione dalla croce, Coro „Tremo la terra“. In Sammlung vorzüglicher Gesangstücke [...], hg. v. Friedrich ROCHLITZ. Bd. 2, Mainz - Paris - Antwerpen: B. Schott's Söhne o. J. [zwischen 1838 und 1840], S. 149-152. Die Vorlage dazu findet sich in D-LEb, Go. S. 472 (Sammlung Gorke). Im selben Band veröffentlichte Rochlitz auch das „Domine Jesu Christe“ aus Fux' Requiem WV IV.111; s. ibid., S. 144-148. Für Näheres zur Sammlung vorzüglicher Gesangstücke s. EHINGER, Hans. Friedrich Rochlitz als Musikschriftsteller. Leipzig: Breitkopf \& Härtel 1929 (Sammlung musikwissenschaftlicher Einzeldarstellungen 9), S. 49-53.

37 Vgl. dazu KIER, Herfried. Kiesewetters historische Hauskonzerte. Zur Geschichte der kirchenmusikalischen Restauration in Wien. Kirchenmusikalisches Jahrbuch 1968, Jg. 52, S. 95-119: 109, 118; ders., Raphael Georg Kiesewetter (1773-1850). Wegbereiter des musikalischen Historismus. Regensburg: Gustav Bosse 1968 (Studien zur Musikgeschichte des 19. Jahrhunderts 13), S. 180. 
bei K[iesewetter] das Ganze am Gründonnerstag 1823, am Gründonnerstag 1825,

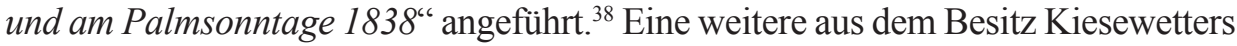
heute in der Musiksammlung der Österreichischen Nationalbibliothek verwahrte Partitur $^{39}$ enthält ein auf der Rückseite des Einbandes eingeklebtes Blatt mit Notizen „zu einer abgekürzten Aufführung des Oratoriums", wobei die mit roter Tinte in die Partitur eingetragenen Streichungen im Vergleich zur anderen Quelle geringfügiger ausgefallen sind. Welcher Aufführung diese Abschrift zuzuordnen ist, bleibt offen. 1839 gab Kiesewetter eine ihm übrige oder erbetene, selbst erstellte und vollständige Kopie an den Sammlerkollegen Fortunato Santini (1778-1861) in Rom weiter, der im Übrigen - im Gegensatz zu Kiesewetter - Abschriften der Madrigale aus La donna forte und Il disfacimento di Sisara besessen hat ${ }^{40}$, und auch Friedrich Rochlitz wurde von Kiesewetter mit der Abschrift von Teilen aus La deposizione $e^{41}$ versorgt. Damit allerdings erschöpt sich die Rezeption des 19. Jahrhunderts. Der Wahrnehmungskreis weitet sich erst, als Arnold Schering in seiner 1911 veröffentlichten Geschichte des Oratoriums mehrere Werke von Fux kurz anspricht. ${ }^{42}$

Kiesewetters Interesse gerade an Fux' La deposizione dalla croce kam - wie ich denke - keineswegs von ungefähr. Unter den Themen fuxscher Oratorien musste eben dieses, eng an das Karfreitagsgeschehen gebundene eine besondere Nähe zur Religiosität des vergangenen Jahrhunderts vermuten lassen - im Sinne eines dem Musikhistoriker Kiesewetter vermutlich vor Augen stehenden historisch gewachsenen Dualismus von Grablegungs- und Auferstehungsfeier, von depositio und elevatio Christi ${ }^{43}$, von Beweinung und Visitatio sepulchri. Von den beiden zentralen Merkmalen, die das Werk als ein für den Wiener Hof verfasstes kennzeichnen ${ }^{44}$, ist nur Traditionstiefe verblieben, Exklusivität dagegen verloren gegangen. Zudem gilt nunmehr, im Gefolge der Aufklärung, ein religiöses Verständnis, das sich für das österliche Geschehen am Glaubensbekenntnis orientiert und den Abschnitt „Crucifixus etiam pro nobis sub Pontio Pilato passus et sepultus est. Et resurrexit tertia die secundam scripturas" zu einer zeitlichen Klammer geraten lässt. Hierin vermute ich einen der Gründe, warum Rochlitz - der einst ein Studium der evangelischen Theologie begonnen hatte ${ }^{45}$ - in seine Sammlung vorzüglicher Gesangstücke eben auch zwei Nummern aus La deposizione auf-

A-Wn, Fonds Kiesewetter, SA. 68. D. 104, genannt bei KIESEWETTER, Raphael Georg. Galerie der alten Contrapunctisten. Wien 1847, S. 26.

A-Wn, Fonds Kiesewetter, SA. 68. B. 21.

Heute D-MÜp, Sant. Hs. 1554, die Madrigale ibid., Sant. Hs. 1555 [1] bzw. [2].

Introduzione und Chor. Heute D-LEb, Go. S. 472 (Sammlung Gorke).

La fede sacrilega, Il fonte della salute, Cristo nell'orto, La cena del Signore und La deposizione dalla croce; s. SCHERING, Arnold. Geschichte des Oratoriums. Leipzig: Breitkopf \& Härtel 1911 (Kleine Handbücher der Musikgeschichte nach Gattungen 3), S. $204 f$.

TRUMMER (wie Anm. 3), S. XVII.

Vgl. GRUBER (wie Anm. 9), S. 3.

EHINGER (wie Anm. 36), S. 3 f. 
nahm, einen zweiten indes darin, dass der Chor „Tremo la terra“ tonmalerisch das Erdbeben nach Jesu Tod nachzeichnet. Damit zog Rochlitz freilich einen Ausschnitt heran, der mit seiner bildhaften Metaphorik in konventionellen Stilmitteln gerade nicht als typisch für Fux' Kompositionsverfahren gelten kann.

Noch eine weitere kontextuelle Veränderung lässt sich aufdecken: Es würde Kiesewetter und Rochlitz wohl erstaunt haben zu erfahren, dass beide Aufführungen von Fux's La deposizione dalla croce am Wiener Hof, sowohl 1728 als auch 1738, nicht am Karfreitag stattfanden, sondern jeweils am Dienstag der Karwoche ${ }^{46}$ Dadurch erstand ein thematischer Zusammenhang zu der am Vormittag jedes Dienstags der Karwoche stattfindenden Kreuzwegprozession vom Stephansdom zur Paulinerkirche in Hernals, wobei - gutes Wetter vorausgesetzt - Kaiser wie gesamter Hofstaat zu Fuß gingen und bei den einzelnen Stationen zum Gebet verweilten. Auch die Hofmusikkapelle war mit unterwegs und musizierte sodann beim abschließenden Hochamt. Ehe der Kaiser mit der Kutsche zurückkehrte, hörte er noch eine stille Messe vor dem Heiligen Grab. ${ }^{47}$ Wieder in der Residenz, erwartete ihn das spätnachmittägliche Sepolcro in der Hofburgkapelle. Beispielhaft überlagern sich in diesem Tagesverlauf Motive und Elemente der Religiositäts-, Mentalitäts- und Musikgeschichte, zu deren Wechselwirkungen viele Einsichten erst noch gewonnen werden wollen.

\begin{tabular}{|c|c|c|c|c|c|c|}
\hline Werk & $\begin{array}{c}\text { erste } \\
\text { (zweite) } \\
\text { Aufführung }\end{array}$ & Librettist & Sujet & $\begin{array}{c}\text { besondere } \\
\text { Funktion }\end{array}$ & \begin{tabular}{|c|} 
musikalische \\
Quellen des 18. / \\
19. Jahrhunderts
\end{tabular} & Textdrucke \\
\hline $\begin{array}{l}\text { Santa } \\
\text { Dimpna, } \\
\text { infanta } \\
\text { d'Irlanda }\end{array}$ & 1702 & \begin{tabular}{|c|} 
Lorenzani \\
(?)
\end{tabular} & hagiographisch & & A-Wn (nur 2. Teil) & $\begin{array}{c}\text { ital. Wien } \\
1702, \\
\text { dt. Wien } 1702\end{array}$ \\
\hline $\begin{array}{l}\text { La Regina } \\
\text { Saba }\end{array}$ & 1705 & Ruggieri & alttestamentlich & & D-SWI & $\begin{array}{c}\text { ital. Wien } \\
1705, \\
\text { dt. Wien } 1705\end{array}$ \\
\hline $\begin{array}{l}\text { La fede } \\
\text { sacrilega } \\
\text { nella morte } \\
\text { del precursor } \\
\text { S. Giovanni } \\
\text { Battista }\end{array}$ & 1714 & Pariati & neutestamentlich & & $\begin{array}{c}\text { A-Wn, } \\
\text { A-KR (nur } \\
\text { Sinfonia), } \\
\text { A-Wsp (nur } \\
\text { Sinfonia, Verlust), } \\
\text { CZ-Pkřiž (Verlust) }\end{array}$ & $\begin{array}{c}\text { ital. Wien } \\
1714\end{array}$ \\
\hline $\begin{array}{l}\text { La donna } \\
\text { forte nella } \\
\text { madre } \\
\text { de' Sette } \\
\text { Maccabei }\end{array}$ & $\begin{array}{c}1715 \\
(1718)\end{array}$ & Pariati & alttestamentlich & & $\begin{array}{c}\text { A-Wn, A-Wgm, } \\
\text { US-NYp } \\
\text { (Madrigale), } \\
\text { D-B (Madrigale), } \\
\text { D-MÜp } \\
\text { (Madrigale), } \\
\text { CZ-Pkřiž (Verlust) }\end{array}$ & $\begin{array}{c}\text { ital. Wien } \\
1715\end{array}$ \\
\hline
\end{tabular}

47 RIEDEL, Friedrich Wilhelm. Kirchenmusik am Hofe Karls VI. (1711-1740). Untersuchungen zum Verhältnis von Zeremoniell und musikalischem Stil im Barockzeitalter. München - Salzburg: Katzbichler 1977 (Studien zur Landes- und Sozialgeschichte der Musik 1), S. 27, 54f. 


\begin{tabular}{|c|c|c|c|c|c|c|}
\hline Werk & $\begin{array}{c}\text { erste } \\
\text { (zweite) } \\
\text { Aufführung }\end{array}$ & Librettist & Sujet & $\begin{array}{l}\text { besondere } \\
\text { Funktion }\end{array}$ & \begin{tabular}{|c|} 
musikalische \\
Quellen des 18. / \\
19. Jahrhunderts
\end{tabular} & Textdrucke \\
\hline $\begin{array}{l}\text { Il trionfo della } \\
\text { Fede }\end{array}$ & 1716 & Maddali & allegorisch & & $A-W n$ & $\begin{array}{c}\text { ital. Wien } \\
1716 \\
\end{array}$ \\
\hline $\begin{array}{l}\text { Il fonte della } \\
\text { salute aperto } \\
\text { dalla Grazia } \\
\text { nel Calvario }\end{array}$ & $\begin{array}{c}1716 \\
(1721)\end{array}$ & Pariati & allegorisch & Sepolcro & $\begin{array}{c}\text { A-Wn, D-MElr, } \\
\text { CZ-Pkřriž (Verlust) }\end{array}$ & $\begin{array}{c}\text { ital. Wien } \\
1716, \\
\text { ital. Wien } \\
1721\end{array}$ \\
\hline $\begin{array}{l}\text { II } \\
\text { disfacimento } \\
\text { di Sisara }\end{array}$ & 1717 & $?$ & alttestamentlich & & \begin{tabular}{|c|} 
A-Wn, \\
US-NYp \\
(Madrigale), \\
D-B (Madrigale), \\
D-MÜp \\
(Madrigale), \\
CZ-Pkřiž (Verlust) \\
\end{tabular} & $\begin{array}{l}\text { ital. Wien } \\
1717\end{array}$ \\
\hline $\begin{array}{l}\text { Cristo } \\
\text { nell'orto }\end{array}$ & $\begin{array}{l}1718 \\
(1723)\end{array}$ & Pariati & neutestamentlich & Sepolcro & $\begin{array}{c}\text { A-Wn, A-Wgm, } \\
\text { A-Sm, D-B, } \\
\text { CZ-Pkřiž (Verlust) }\end{array}$ & $\begin{array}{l}\text { ital. Wien } \\
1718, \\
\text { ital. Wien } \\
1721, \\
\text { ital. Brünn } \\
1731\end{array}$ \\
\hline $\begin{array}{l}\text { Gesù Cristo } \\
\text { negato da } \\
\text { Pietro }\end{array}$ & 1719 & Pariati & neutestamentlich & Sepolcro & $\begin{array}{c}\text { A-Wn, A-Wgm, } \\
\text { CZ-Pkřiž (Verlust) }\end{array}$ & $\begin{array}{c}\text { ital. Wien } \\
1719, \\
\text { ital. Prag } 1750\end{array}$ \\
\hline $\begin{array}{l}\text { La cena del } \\
\text { Signore }\end{array}$ & 1720 & Pariati & neutestamentlich & Sepolcro & A-Wn, A-Wgm & $\begin{array}{c}\text { ital. Wien } \\
1720\end{array}$ \\
\hline $\begin{array}{l}\text { Il testamento } \\
\text { di nostro } \\
\text { Signor Gesù } \\
\text { Cristo sul } \\
\text { Calvario }\end{array}$ & $\begin{array}{c}1726 \\
(1727)\end{array}$ & Pariati & halballegorisch & Sepolcro & $\begin{array}{l}\text { A-Wn, A-Wgm, } \\
\text { D-MElr, } \\
\text { A-Wn (einzelne } \\
\text { Arien), } \\
\text { GB-Lbl (einzelne } \\
\text { Arien) }\end{array}$ & $\begin{array}{l}\text { ital. Wien } \\
1726, \\
\text { ital. Wien } \\
1727\end{array}$ \\
\hline $\begin{array}{l}\text { La } \\
\text { deposizione } \\
\text { dalla croce di } \\
\text { Gesù Cristo } \\
\text { Salvator } \\
\text { nostro }\end{array}$ & $\begin{array}{c}1728 \\
(1738)\end{array}$ & Pasquini & neutestamentlich & Sepolcro & $\begin{array}{c}\text { A-Wn, A-Wgm, } \\
\text { D-WD, } \\
\text { A-Sm, A-HE, } \\
\text { D-MEIr, D-B, } \\
\text { A-Wn (einzelne } \\
\text { Arien), } \\
\text { A-Wn (Fonds } \\
\text { Kiesewetter), } \\
\text { D-MÜp, } \\
\text { D-LEb (Einltg. } \\
\text { und Chor) }\end{array}$ & $\begin{array}{c}\text { ital. Wien } \\
1728, \\
\text { ital. Brünn } \\
1729, \\
\text { ital. Wien } \\
1738, \\
\text { ital. Arezzo } \\
1751, \\
\text { ital. Prag } \\
1751, \\
\text { ital. Rom 1764 }\end{array}$ \\
\hline
\end{tabular}

Tab. 1: Übersicht zu den Oratorien von Johann Joseph Fux für den Wiener kaiserlichen Hof

Thomas Hochradner (thomas.hochradner@moz.ac.at), Universität Mozarteum Salzburg, Abteilung für Musikwissenschaft. 


\section{ABSTRACT \\ WITH A SLIGHT ECHO. ON THE RECEPTION HISTORY OF JOHANN JOSEPH FUX'S ORATORIOS}

There is no doubt that Johann Joseph Fux created his oratorios (with the exception perhaps of a „Oratorium germanicum“ attributed to Fux which survived in the Kremsmünster monastery) for performances during season of lent at the Viennese imperial court during the presence of the Hofburg ensemble. But the oratorios of the imperial court music director are taking a special position within the relatively hermetic inventory of the imperial ensemble. For their performances in other places, including Brno and Prague, were more common than performances of his secular dramatic works, transcriptions of oratorios belonged also in the later 18th and 19th centuries to those few Fux's compositions, which attracted the attention of reception history. The contexts of this interest nevertheless changed and they can be identified as religiosity, mentality and music historical consciousness which newly create its frame.

\section{Key words}

oratorio, Johann Joseph Fux, reception history

\section{Bibliography}

BACCIAGAlUPPI, Claudio. Rom, Prag, Dresden. Pergolesi und die Neapolitanische Messe in Europa. Kassel et al.: Bärenreiter 2010 (Schweizer Beiträge zur Musikforschung 14).

BENNETT, Lawrence E. A little-known Collection of early-eighteenth-century Vocal Music at Schloss Elisabethenburg, Meiningen. Fontes Artis Musicae 2001, Jg. 48, S. 250-302.

CORETH, Anna. Pietas Austriaca. Österreichische Frömmigkeit im Barock. 2. Aufl. Wien: Verlag für Geschichte und Politik 1982 (Österreich-Archiv. Schriftenreihe des Instituts für Österreichkunde o. Zl.).

EHINGER, Hans. Friedrich Rochlitz als Musikschriftsteller. Leipzig: Breitkopf \& Härtel 1929 (Sammlung musikwissenschaftlicher Einzeldarstellungen 9).

FLOTZINGER, Rudolf. Das `Oratorium Germanicum de Passione` von Johann Joseph Fux. Schritte der Annäherung an Autoren- und Gattungsfragen. In Festschrift Hellmut Federhofer zum 100. Geburtstag, hg. v. Axel BEER in Verb. mit Gernot GRUBER und Herbert SCHNEIDER. Tutzing: Schneider 2011 (Mainzer Studien zur Musikwissenschaft 45), S. 91-105.

FUKAČ, Jiř́. Die Oratorienaufführungen bei den Prager Kreuzherren mit dem Roten Stern als Typ lokaler Musikfeste. Sborník praci filozofické fakulty Brněnské univerzity 1994, Jg. 29, S. 69-89.

FUKAČ, Jiř́. Pöltenberg, Wien und die Musikkultur der Prager Kreuzherren. In Österreichische Musik-Musik in Österreich. Beiträge zur Musikgeschichte Mitteleuropas. Theophil Antonicek zum 60. Geburtstag, hg. v. Elisabeth Theresia HILSCHER. Tutzing: Schneider 1998 (Wiener Veröffentlichungen zur Musikwissenschaft 34), S. 171-177.

GRONDA, Giovanna (Hg.). La carriera di un librettista. Pietro Pariati da Reggio di Lombardia. Reggio Emilia: Il Mulino 1990 (Proscenio. Quaderni del Teatro Municipale »Romolo Valli« di Reggio Emilia 5).

GRUBER, Gernot. Das Wiener Sepolcro und Johann Joseph Fux. 1. Teil [ein zweiter Teil ist nicht erschienen]. Graz: Johann-Joseph-Fux-Gesellschaft 1972 (Jahresgabe der Johann-Joseph-Fux-Gesellschaft 9).

HILSCHER, Elisabeth Theresia. Der Kaiserliche Hofpoet Pietro Metastasio (1698-1782). Zur Kulturgeschichte des Hofes Kaiser Karls VI. Graz: Johann-Joseph-Fux-Gesellschaft 2000 (Jahresgabe der Johann-Joseph-Fux-Gesellschaft 23). 
HOCHRADNER, Thomas -VÖRÖSMARTY, Géza Michael. Zur Musikpflege am Altar Mária Pócs (Maria Pötsch) in St. Stephan in Wien. Studia Musicologica Academiae Scientiarum Hungaricae 2000, Jg. 41, Heft 1-3, S. 133-175.

KADE, Otto. Die Musikalien-Sammlung des Großherzoglichen Mecklenburg-Schweriner Fürstenhauses. 2 Bände, Schwerin 1893, Nachdruck Hildesheim - New York: Olms 1974.

KIER, Herfried. Kiesewetters historische Hauskonzerte. Zur Geschichte der kirchenmusikalischen Restauration in Wien. Kirchenmusikalisches Jahrbuch 1968, Jg. 52, S. 95-119.

KIER, Herfried. Raphael Georg Kiesewetter (1773-1850). Wegbereiter des musikalischen Historismus. Regensburg: Gustav Bosse 1968 (Studien zur Musikgeschichte des 19. Jahrhunderts 13).

MRAZ, Gerda. Die Kaiserinnen aus dem Welfenhaus und ihr Einfluß auf das geistig-kulturelle Leben in Wien. In Johann Joseph Fux und seine Zeit. Kultur, Kunst und Musik im Spätbarock, hg. v. Arnfried EDLER und Friedrich Wilhelm RIEDEL. Laaber: Laaber 1996 (Publikationen der Hochschule für Musik und Theater Hannover 7), S. 75-91.

PETERMAYR, Klaus. Fux oder Caldara? - zum wiederaufgefundenen 〉Oratorium Germanicum (E 61). Auf Fux-Jagd 2004, Jg. 12, Nr. 1/2, S. 23-32.

PETERMAYR, Klaus. Das Oratorium Germanicum (E 61) von Johann Joseph Fux. Jahrbuch des rism-österreich 2011, Jg. 2 (Veröffentlichungen des rism-österreich A 16), S. 79-91.

RIEDEL, Friedrich Wilhelm. Kirchenmusik am Hofe Karls VI. (1711-1740). Untersuchungen zum Verhältnis von Zeremoniell und musikalischem Stil im Barockzeitalter. München - Salzburg: Katzbichler 1977 (Studien zur Landes- und Sozialgeschichte der Musik 1).

SCHERING, Arnold. Geschichte des Oratoriums. Leipzig: Breitkopf \& Härtel 1911 (Kleine Handbücher der Musikgeschichte nach Gattungen 3).

SCHINDLECKER, Katrin. Musik in der Osterliturgie im 20. Jahrhundert. Eine generelle Beobachtung mit zwei Fallbeispielen aus der heutigen Zeit. Diplomarbeit Universität Mozarteum Salzburg 2008.

TRUMMER, Johann. Das Heilige Grab und die Sepolcro-Komposition. In Johann Joseph Fux: Il fonte della salute, vorgelegt von Martin JIRA, Graz: ADEVA 2008 (Johann Joseph Fux. Sämtliche Werke IV/5), S. XV-XXIII. 
